\title{
Evaluation of HIV prevention programmes: the case of Avahan
}

\author{
Stefano M Bertozzi, ${ }^{1}$ Nancy Padian, ${ }^{2}$ Tyler E Martz ${ }^{1}$
}

Current calls to action for HIV prevention programming stress the need for more strategic, combination prevention strategies along with evaluation components to measure effectively the impacts of such efforts. The Avahan project, implemented since 2003, is one example of a combination prevention programme for a concentrated epidemic that offers a clear example of what can be achieved when a programme is based on significant 'know your epidemic' research before implementation, and when data are used to optimise a delivery programme to maximise coverage of services. The rapid scale-up, the documentation of quality and coverage indicators, and perhaps most importantly, the application of modern, data-driven management tools to the delivery of a largescale public health programme, are Avahan's most exemplary qualities.

Avahan is surely the world's largest HIV prevention programme in a country with a concentrated epidemic and perhaps the largest anywhere. It focuses on reaching the populations key to the spread of HIV in India: sex workers and their clients, men who have sex with men (MSM) and drug users. The team's experience with management information systems allowed them to monitor closely the implementation of services and in close to real-time adjust their practices to improve the quality and coverage of their services.

In addition to the 'know your epidemic' mapping of populations at risk, Avahan used market research to inform the design of their service delivery, ensuring that the facilities were conveniently located, that they were responsive to the specific needs

${ }^{1}$ Instituto Nacional de Salud Publica, Av. Universidad, Cuernavaca, Morelos, Mexico ${ }^{2}$ University of California, Berkeley, California, USA

Correspondence to Dr Stefano M Bertozzi, Instituto Nacional de Salud Publica, Av Universidad 655, Cuernavaca, Morelos, Mexico;

bertozzi@alum.mit.edu

\section{UNIOCKED}

This paper is freely available online under the BMJ Journals unlocked scheme, see http://sti.bmj.com/site/ about/unlocked.xhtml of the population being served, etc. Had they been selling shoes or software, they would have been able to measure their success from the day they opened for business based on revenues minus expenses. Unfortunately, however, the connection between sales of HIV prevention services (coverage and uptake) and revenue (HIV infections averted) is far less clear. As a result, it is not obvious how much of the apparent decline in HIV prevalence in the states where the programme operated can be attributed to the Avahan programme. Although it seems reasonable to assume that the massive scale-up in delivery of prevention services played an important role in contributing to the decline in prevalence, the apparent timing of the decline has raised questions about whether the decline was occurring independent of the programme efforts. ${ }^{1}$ The likely effectiveness of behavioural HIV prevention interventions when implemented in various contexts is difficult to predict. A programme manager cannot assume that a package of 'best practice' interventions derived from other settings will result in reduced HIV incidence in his/ her context. This is further complicated by the fact that there are often scarce efficacy/ effectiveness data on some interventions in any setting. Given these uncertainties, it is essential that large programmes such as Avahan collect data that permit rigorous assessment of the impact on HIV incidence. If such data can be collected as the programme is implemented from different sites (preferably randomly determined), which receive different variations of the

Coverage and uptake: If a programme cannot achieve sufficient coverage or the target population is unwilling to participate, or if there is little uptake, then successful outcomes are unlikely. Evaluating these components may reveal that coverage was poor as a result of inefficient implementation or that poor uptake was linked to culturally inappropriate content etc. Verma et $a^{2}$ described methods for assessing the scale-up and coverage of large-scale HIV prevention services provided to female sex workers (FSW) and high-risk MSM during the first 5 years of Avahan. Results revealed impressively high levels of coverage as verified by survey data after 60 months: $75 \%$ of the denominator intended to be covered (69 districts) was met monthly; $85 \%$ FSW and $64 \%$ high-risk MSM ever contacted had used sexually transmitted infection services at least once.

By using data on condom sales and distribution data from programmes and the private sector, as well as sexual behaviour data obtained from general population surveys, Bradley et al ${ }^{3}$ assessed the proportion of FSW who used condoms with clients and other partners. Here they combined empirical data with modelling techniques that permitted comparisons of scenarios that discounted the number of condoms that might have been purchased for non-commercial sex. These data should be helpful in validating actual condom use when triangulated with other available data, such as self-reported condom use.

Finally, Blankenship et al ${ }^{4}$ assessed programme uptake by examining predictors of participation among FSW by using two rounds of serial cross-sectional surveys. Awareness of and participation in the programme increased among women who were publicly willing to admit that they were sex workers.

Quality and fidelity of interventions: Mogasale et $a l^{5}$ assessed the overall quality of coverage (towards all targeted risk groups) on a routine quarterly basis using an 80 question survey to assess the coverage, quality of services, use of referral networks, community involvement and technical support. This routine quantitative score revealed a significant improvement in programme delivery over time. Two other papers examined the association between coverage and behaviour change.

Ramakrishnan et $a l^{6}$ measured coverage by assessing whether FSW received the three core services of the programme coverage, and then examined whether this coverage was associated with knowledge and use of condoms. Lipovsek et al, ${ }^{7}$ who examined coverage levels from the behaviour change communication component of the programme in association with consistent condom use among male clients of FSW, determined that exposure to the programme had positive effects on these outcomes. 
Effectiveness: Alery et a ${ }^{8}$ examined the association between prevalent infections among sex workers and their clients and antenatal care HIV (ANC) prevalence as a way to examine the contribution of commercial sex to infection in the general population. This cross-sectional assessment was conducted towards the end of the programme. Results confirmed that infection rates among FSW were positively associated with the ANC infection rate, which validated the targeting of the Avahan programme and indicated the need for continued focus in this group.

Pickles et $a l^{9}$ confirmed the findings of Alery et al ${ }^{8}$ using a deterministic compartmental HIV/STI transmission model parameterised using data from Belgaum and Mysore districts in south India. Multiple model fits to STI/HIV data from FSW examined whether all prevalent HIV/herpes simplex virus type 2 infections in each district could be accounted for solely by HIV/herpes simplex virus type 2 transmission between FSW/clients, and to their non-commercial partners. Although uncertainty about client population size reduced model accuracy, they nevertheless concluded that for men, most HIV infections were caused by sex with FSW, whereas for women most infections are bridging infections from their male partners who are also clients of FSW.

Finally, Ramesh et $a{ }^{10}$ came closest to examining the impact of Avahan on FSW using dynamic transmission models. They used prevalence from two cross-sectional surveys of randomly sampled FSW in five Karnataka districts to assess changes over time in STI/HIV prevalence, condom use and HIV prevention programme exposure. The survey was conducted in 2004 and 2005 and then again 27-37 months later. They also assessed exposure to the programme by asking if the FSW had been contacted by a peer educator. The results indicated a significant decrease in HIV, syphilis, gonorrhoea and chlamydia, and increased condom use with regular clients.

programme (varied by timing, intensity, or composition/character of the components of the programme), then it will be possible to measure impact, and (if successful) to make the case convincingly both for further financial support and for adapting/ expanding the programme to other settings. It is also possible for the evaluation design to provide feedback long before implementation is complete, so that programmes can make changes to improve effectiveness on an ongoing basis.

The papers included in this special issue document the broad range of monitoring and evaluation activities that have accompanied the implementation of the Avahan programme, highlight some of the evaluation lessons learnt and provide an overview of the approaches that the different papers have taken to the evaluation of Avahan.

Although Avahan provides many examples of quality evaluation, had they built a prospective, more robust evaluation of impact into its implementation, they could have more convincingly demonstrated effectiveness. The impact of Avahan has been assessed through repeat cross-sectional surveys combined with modelling to confirm an effect over time; promising and highly suggestive, but not definitive. With regard to process indicators such as coverage, quality and tailoring of services, Avahan is unsurpassed; they were able to fine-tune the programme with regard to such outcomes on an ongoing basis. However, with regard to impact, might Avahan represent a missed opportunity? For example, it is unclear why they could not have implemented the programme in a randomised phased fashion (eg, stepped wedge design). They could also have included a cohort to measure incidence directly, more comprehensive baseline data, or control communities. Had Avahan used an adaptive design that permitted early peeks at outcomes, they would have been able to tailor the programme based on trends in impact (in addition to trends in coverage and quality) further optimising the likelihood of achieving the greatest impact.

\section{Competing interests None.}

Provenance and peer review Commissioned; not externally peer reviewed.

Accepted 22 October 2009

Sex Transm Infect 2010:86:i4-i5. doi:10.1136/sti.2009.039263

\section{REFERENCES}

1. Arora $\mathbf{P}$, Kumar $\mathrm{R}$, Bhattacharya $\mathrm{M}$, et al. Trends in HIV incidence in India from 2000 to 2007. Lancet 2008;372:289-90.

2. Verma R, Shekhar A, Khobragade S, et al. Scale-up and coverage of Avahan: a large-scale HIV-prevention programme among female sex workers and men who
Costs and cost-effectiveness:

Chandrashekar et $a^{11}$ presented excellent monitoring of costing data and noted that during the rapid scale-up of the programme, a significant reduction in average costs of the programme was observed between start-up and the first 2 years of implementation. Furthermore, large-scale interventions should quantify scale effects when planning for future resource requirements.

have sex with men in four Indian states. Sex Transm Infect 2010;86:176-82

3. Bradley J, Moses S, Blanchard JF, et al. Assessing reported condom use among female sex workers in southern India through examination of condom availability. Sex Transm Infect 2010;86 i44-8.

4. Blankenship KM, Burroway R, Reed E. Factors associated with awareness and utilisation of a community mobilisation intervention for female sex workers in Andhra Pradesh, India. Sex Transm Infect 2010;86:i69-75

5. Mogasale V, Wi TC, Das A, et al. Quality assurance and quality improvement using supportive supervision in a large-scale STI intervention with sex workers, men who have sex with men/transgenders and injecting-drug users in India. Sex Transm Infect 2010;86:i83-8

6. Ramakrishnan L, Gautam A, Goswami $\mathrm{P}$, et al. Programme coverage, condom use and STI treatment among FSWs in a large-scale HIV prevention programme: results from cross-sectional surveys in 22 districts in southern India. Sex Transm Infect 2010;86 i62-8.

7. Lipovsek V, Mukherjee A, Navin D, et al. Increases in self-reported consistent condom use among male clients of female sex workers following exposure to an integrated behaviour change programme in four states in southern India. Sex Transm Infect 2010;86 i25-32.

8. Alary $\mathbf{M}$, Jayachandran AA, Lowndes $\mathrm{CM}$, et al. Ecological analysis of the association between highrisk population parameters and HIV prevalence among pregnant women enrolled in sentinel surveillance in four southern India states. Sex Transm Infect 2010;86:110-16

9. Pickles M, Foss AM, Vickerman P, et al. Interim modelling analysis to validate reported increases in condom use and assess HIV infections averted among female sex workers and clients in southern India following a targeted HIV prevention programme. Sex Transm Infect 2010;86 i33-43.

10. Ramesh BM, Beattie TSH, Shajy I, et al. Changes in risk behaviours and prevalence of sexually transmitted infections following HIV preventive interventions among female sex workers in five districts in Karnataka state, south India. Sex Transm Infect 2010:86:117-24.

11. Chandrashekar S, Guinness L, Kumaranayake L, et al. The effects of scale on the costs of targeted HIV prevention interventions among female and male sex workers, men who have sex with men and transgenders in India. Sex Transm Infect 2010;86: i89-94. 


\section{Key messages}

- International backpackers attending the clinic had higher rates of genital chlamydial infection than a local comparison population.

- International backpackers have high rates of partner change, hazardous levels of alcohol consumption and low rates of condom usage, creating an environment conducive to STI transmission.

- Backpackers should be a priority population for sexual health promotion and access to services.

Acknowledgements The authors would like to thank Heng Lu and Ben Judd for data management.

\section{Competing interests None.}

Ethics approval This study was conducted with the approval of the South Eastern Sydney Illawarra Area Health Service Ethics Committee.

Contributors CE developed the study idea and assisted with study design; HW analysed the data; AMM designed the study and wrote the manuscript; BD designed the study and edited the manuscript. All authors reviewed the study manuscript.

Provenance and peer review Not commissioned; externally peer reviewed.

\section{REFERENCES}

1. Hawkes S, Hart G, Bletsoe E, et al. Risk behaviour and STD acquisition in genitourinary clinic attenders who have traveled. Genitourin Med 1995;71:351-4.

2. Melbye $\mathbf{M}$, Biggar R. A profile of HIV-risk behaviours among travellers-a population based study of Danes visiting Greenland. Scand J Soc Med 1994;22:204-8.

3. Rogstad K. Sex, sun, sex and STIs: sexually transmitted infections acquired on holiday. BMJ 2004;329:214-17.
4. Richens J. Sexually transmitted infections and HIV among travellers: a review. Travel Med Infect Dis 2006;4:184-95.

5. Mercer C, Fenton K, Wellings K, et al. Sex partner acquisition while overseas: results from a British national probability survey. Sex Transm Infect 2007;83:517-22.

6. Hughes K, Downing J, Bellis $\mathrm{M}$, et al. The sexual behaviour of British backpackers in Australia. Sex Transm Infect 2009:85:477-82.

7. Cabada M, Echevarria J, Seas C, et al. Sexual behaviour of international travelers visiting Peru. Sex Transm Dis 2002;29:510-13.

8. Bloor M, Thomas M, Hood $\mathrm{K}$, et al. Differences in sexual risk behaviour between young men and women travelling abroad from the UK. Lancet 1998;352:1664-8.

9. Egan C. The sexual behaviours, condom use and factors influencing casual sex among backpackers and other international travellers. Can J Hum Sex 2001;10:41-58.

10. Tourism Research Australia. Backpackers in Australia. Belonnen, ACT: Tourism Research Australia, 2008. <http://www.tra.australia.com/content/documents/ Snapshots/2008/BackPacker 07 FINAL.pdf> (accessed 20 0ct 2009).

11. Department of Immigration and Citizenship. Australian Government. Canberra, 2009. http://www.immi.gov.au/visitors/working-holiday/visa-options.htm laccessed 12 June 2009)

12. Yallop S, Murray C, Egan C, et al. Safe in the sack - backpackers' sexual health project. Sydney: FPA Health, 2005.

13. National Health and Medical Research Council. Australian Alcohol Guidelines: Health risks and benefits, 2001. http://www.nhmrc.gov.au/publications/synopses/ ds9syn.htm (accessed 20 0ct 2009).

14. Arvidson M, Hellberg D, Mardh PA. Sexual risk behavior and history of sexually transmitted diseases in relation to casual travel sex during different types of journeys. Acta Obstet Gynecol Scand 1996;75:490-4.

15. Egan C. Sexual behaviour of international backpackers in the context of travel in Australia. PhD thesis. Sydney: Faculty of Arts and Social Sciences, University of New South Wales, 2009.

16. Chen M, Rohrsheim R, Donovan B. Chlamydia trachomatis infection in Sydney women. Aust NZ J Obstet Gynaecol 2005;45:410-13.

17. Carter S, Horn K, Hart G, et al. The sexual behaviour of international travelers at two Glasgow GUM clinics. Int J STD AIDS 1997;8:336-8.

18. Mendelsohn R, Astle L, Mann M. Sexual behaviour in travellers abroad attending an inner-city genitourinary medicine clinic. Genitourin Med 1996;72:43-6.

19. Bellis $\mathbf{M}$, Hughes $\mathrm{K}$, Dillon $\mathrm{P}$, et al. Effects of backpacking holidays in Australia alcohol, tobacco and drug use of UK residents. BMC Public Health 2007;7:1.

20. Bellis $\mathbf{M}$, Hughes K, Thomson R, et al. Sexual behaviour of young people in international tourist resorts. Sex Transm Infect 2004;80:43-7.

\section{Corrections}

Hettiarachchi N, Ashbee HR, Wilson JD. Prevalence and management of non-albicans vaginal candidiasis. Sex Transm Infect 2010;86:99-100. The surname of the first author was misspelled. The correct name is Hettiarachchi not Hetticarachchi. The journal apologises for this error.

Sex Transm Infect 2010;86:250. doi:10.1136/sti.2009.040386corr1

Jurstrand M, Christerson L, Klint M, et al. Characterisation of Chlamydia trachomatis by ompA sequencing and multilocus sequence typing in a Swedish county before and after identification of the new variant. Sex Transm Infect 2010;86:56-60. There was an error in the order of the references. The corrected version is available online.

Sex Transm Infect 2010;86:250. doi:10.1136/sti.2009.037572corr1

Bertozzi SM, Padian N, Martz TE. Evaluation of HIV prevention programmes: the case of Avahan. Sex Transm Infect 2010;86:i4-5. There were several errors in this editorial. Reference 9 referred to an unpublished paper by Vickerman, not to the paper by Pickles, which was published in the same supplement.

Secondly, the statement "Finally Ramesh et al came closest to examining the impact of Avahan on FSW using dynamic transmission models. They used prevalence from two crosssectional surveys..." is incorrect. Ramesh does not use a dynamic transmission model-this is referring to one of the two modelling papers. Ramesh uses a time-trend analysis from two cross sectional surveys.

Finally, the name Alary is misspelled Alery twice in the editorial.

The journal apologies for these errors.

Sex Transm Infect 2010;86:250. doi:10.1136/sti.2009.039263corr1

Laga M, Galavotti C, Sundaramon S, et al. The importance of sex-worker interventions: the case of Avahan in India. Sex Transm Infect 2010;86:16-7. The surname of the author Sundar Sundaramon was incorrectly spelt. The correct spelling is Sundararaman. 